\title{
Unified Reliability Index Development for Utility Performance Assessment
}

\author{
Developing a Novel Normalization-Based Index and Comparing it with a Fuzzy Inference \\ Unified Index
}

\author{
Hatem Sindi $^{1}$ (D) Ehab El-Saadany ${ }^{1,2}$
}

Received: 7 January 2016 / Revised: 10 May 2016 / Accepted: 25 May 2016 / Published online: 8 June 2016

(C) Springer Science+Business Media Singapore 2016

\begin{abstract}
The major purpose of the continuous development of reliability indices is to capture a comprehensive view of systems performance. The lack of consensus among utilities and regulators on which indices should be used complicates the problem more. Regulators inevitably make comparisons between utilities' routinely reported reliability indices. Thus, an adequate and fair process needs to be implemented. Utilities are reporting indices in a chaotic way. One utility might report SAIFI and SAIDI, while others report SAIFI and CAIDI. In this work, a development of a unified reliability index, which can yield proper performance assessment, fair comparisons, and reflection of all the knowledge embedded within all current indices, will be formed. The initiated unified index may provide several benefits, among which is suitable standards design, improved tools for planning and design optimization, and less technical burden on operators. Also, the development of a unified reliability index requires the initiation of a standard normalization methodology based on a maximum that takes into account the variation between systems under study. After normalization, combining indices is made possible through a proposed methodology. The developed methodology is validated by comparing it to both simple averaging technique and a fuzzy inference based engine.
\end{abstract}

Keywords Reliability · Unified Index · Fuzzy inference · Unification $\cdot$ Assessment $\cdot$ Utility performance

Hatem Sindi

hsindi@uwaterloo.ca

1 University of Waterloo, 200 University Ave W, Waterloo, ON N2L 3G1, Canada

2 Present Address: Petroleum Institute (PI), Abu Dhabi, UAE

\section{List of Symbols}

\section{Acronyms}

ASAI Average Service Availability Index

ASIDI Average System Interruption Duration Index

ASIFI Average System Interruption Frequency Index

ASUI Average Service Unavailability Index

CAIDI Customer Average Interruption Duration Index

CAIFI Customer Average Interruption Frequency Index

CELID Customers Experiencing Long Interruption Durations

CEMI $_{n}$ Customers Experiencing Multiple Interruptions

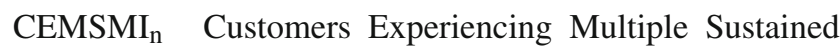
Interruption and Momentary Interruption Events

CTAIDI Customer Total Average Interruption Duration Index

MAIFI $_{E}$ Momentary Average Interruption Event Frequency Index

MAIFI Momentary Average Interruption Frequency Index

SAIDI System Average Interruption Duration Index

SAIFI System Average Interruption Frequency Index UI Unified Index

\section{Variables and Parameters}

CI Customers interrupted

CMI Customer minutes of interruption

$\mathrm{CN}_{\mathrm{k}>\mathrm{n}} \quad$ Customers experienced $\mathrm{n}$ or more sustained interruptions 


\begin{tabular}{|c|c|}
\hline $\mathrm{CN}_{\mathrm{k}>\mathrm{S}}$ & $\begin{array}{l}\text { Customers that experienced } \mathrm{S} \text { or more hours } \\
\text { duration }\end{array}$ \\
\hline $\mathrm{CN}_{\mathrm{k}>\mathrm{T}}$ & $\begin{array}{l}\text { Customers that experienced } \mathrm{T} \text { or more hours } \\
\text { duration }\end{array}$ \\
\hline $\mathrm{CNT}_{\mathrm{k}>\mathrm{n}}$ & $\begin{array}{l}\text { Customers experienced } \mathrm{n} \text { or more sustained } \\
\text { interruptions and momentary interruption } \\
\text { events }\end{array}$ \\
\hline $\mathrm{CN}$ & $\begin{array}{l}\text { Distinct customers experienced a sustained } \\
\text { interruption }\end{array}$ \\
\hline $\mathrm{N}_{\mathrm{T}}$ & Total number of customers served for the area \\
\hline$\lambda_{m \max _{(g)}}$ & Global maximum momentary failure rate \\
\hline$\lambda_{m \max _{(l)}}$ & Local maximum momentary failure rate \\
\hline$\lambda_{s \max _{(g)}}$ & Global maximum sustained failure rate \\
\hline$\lambda_{s \max _{(l)}}$ & Local maximum sustained failure rate \\
\hline$\lambda_{m}$ & Momentary failure rate \\
\hline$\lambda_{s}$ & Sustained failure rate \\
\hline$r_{s \max _{(g)}}$ & Global maximum sustained failure duration \\
\hline$r_{s \max _{(l)}}$ & Local maximum sustained failure duration \\
\hline$r_{s}$ & Sustained failure duration \\
\hline
\end{tabular}

\section{Introduction}

Accommodating increased demand with older techniques involves many technical and non-technical difficulties, such as regulatory, environmental, fuel cost, project cost, and transmission infrastructure. Therefore, regulators recommend several practices to overcome these challenges. Demand side management, sustainable distributed generation, and distribution system reliability enhancements are examples of such recommendations.

The significant point is that neither regulators nor utilities come with a complete understanding of how to improve system reliability. Regulators use utilities' historical data to assess performance, while utilities vary on the perception of historical data. Some consider them guidelines, others consider them goals to achieve, and still others consider them absolute standards. Nevertheless, regulators will inevitably compare and cross-compare performance [10].

Several reliability indices make assessments. There has not been a consensus on which indices should be used. While reliability indices have accommodated development over recent years; these indices do not provide the proper tools to achieve an adequate standard design or objective comparisons. Enhancing performance, penalizing, or awarding different parties in distribution systems requires adequate, simple, and accurate assessment.

Following customer demands for a more reliable service, and steps toward a smart distribution system, better tools to assess and enhance system performance should be targeted. For instance, tools to collect, analyze and act upon system data must be developed in order to reach smart grid technology. These tools require faster, accurate, and impartial techniques. Current methodologies, although usage varies between utilities and regulators, have an embedded bias in the output of assessment for systems reliability. Comparisons cannot be accurately and impartially conducted due to several reasons, among which are deciding on a broad range of metrics, system topology variation, type of customer, perception of these metrics, and technical background requirements.

\section{Distribution System Reliability Indices}

People have been coping with reliability problems in their homes, offices, factories, and a variety of other settings. People do not always seem to mind a weak system configuration that promotes lower electricity prices, yet some customers, especially those in the commercial and industrial sectors of the system, require certain availability levels. Utilities and regulators have always impacted distribution system reliability. However, attention paid to this area has been significantly less than a generation regarding reliability studies [8]. These studies are mainly concerned with modeling and evaluation. Nonetheless, attention was given even before any form of practical model experience. This attention began to change in the 1960s, after developing failure rates and the introduction of the Markov process in reliability studies [7,18,28]. For reliability studies in distribution systems, some metrics was developed to conduct further analysis. These metrics differ in their hierarchal levels within the distribution network. Some provide information about the distribution network as a whole; others reflect the performance of specific parts, such as feeders, load point, or the collected part of the system. However, further studies explore the new concepts introduced in systems, such as introducing distributed generation and new regulations that require new studies to be conducted [12].

It is important to highlight the need for advanced tools in the new paradigm (i.e. smart grids), as that is the scope of this work. These tools involve, but are not restricted to, reliability studies. New technologies and implementations in data acquisition, data mining, and analysis are necessary. These technologies are needed to improve tool efficiently; they are also needed due to the unique nature of smart distribution systems. In such systems, new regulations are necessary for reliability, contracts, customer-utility-regulator relationships, and the paradigm shift in thinking of distribution systems as passive. Reliability studies are vital to distribution system studies. One can understand the significance of the amount of literature that has been written on this topic.

From a customer perspective, ease of communication with the utility during an interruption of service, and the time needed to restore the service, are key factors in the assessment of service quality [31]. On the other hand, utilities usually assess the service reliability at load point or 
customer level rather than from the generation or transmission. Nevertheless, these concepts, amongst others, can highlight how important reliability is both for clients and utilities.

Utilities invest large amounts of money to upgrade, build, or maintain systems. The use of reliability studies, although they do not guarantee global optimality, minimizes losses. The tradeoff between enhancing service quality (reliability) and total cost cannot currently be avoided. Unfortunately, customers tend not to fully understand this compromise [36]. Reliability studies play a vital role in enhancing operational conditions. During restoration and reconfiguration, reliability studies, such as reliability worth or reliability indices, are used [9]. Regulators have also been actively involved in reliability studies [17]. Utilities routinely report reliability data to regulators [15].

While technical advancement is rapidly growing in many aspects of power systems, adequate tools to assess reliability are still necessary. In a general sense, reliability metrics (indices) were developed to reflect system performance in a scientific manner. Consequently, additional benefits have been derived from these indices. Although in distribution systems, the methodology includes starting with basic components, then aggregating different probabilities to arrive at an average number, the derived number is only partially reflective of the reliability of the system.

The adoption of the IEEE Std 1366-2012 and the IEEE 2.5 beta methodology that classifies normal daily operational reliability data and major events data is highly recommended by [20]. These indices can be categorized into two major sections: Load Point and System Indices. The work in [16] showcase a utility report utilizing the aforementioned recommendations. Almost all indices are derived from customer information systems (CIS); therefore, averaging is used in calculating the indices, due to ease of access to customer data. According to [35], utilities are continuing to understand the need for more than one or two indices to capture service quality and to design an effective implementation plan accordingly.

For load point indices, three main indices are commonly used in load point reliability metrics [21]. These indices characterize: first, the frequency of interruption the load point has suffered over the study period; second, the average outage time for each interruption over the study period; lastly, the average time of unavailability for load points due to all interruptions suffered over the reporting period $[10,15]$. Although the three indices have been heavily studied for improving the accuracy of their calculation, they are still predictive [33]. They are predictive rather than deterministic because they are composed of aggregated averages that directly depend on several probabilities [1].

The system performance can also be assessed on an overall system basis. The indices reflect the adequacy of the overall system supply and indicate system behavior and response. According to [20], 14 indices are recommended for assessing system reliability performance. Some of these indices were developed as early as the 1960 s $[5,13,18,28]$. Some other indices were introduced more recently $[6,14]$.

In numerous reliability surveys, the general decision was that utilities are increasingly interested in incorporating more indices [3,11]. Moreover, comparison and cross-comparison of reported data amongst utilities becomes inevitable for regulators [15]. However, the current infrastructure of indices does not promote fair and accurate comparison. Authors in [30] studied the impact of momentary and sustained interruptions in the design process. They concluded that momentary interruptions were as important as sustained ones when it comes to reliability-based distribution system design. Moreover, this, among other reasons, is a push toward system design based on reliability studies. The more reliability indices are included, the more comprehensive the study becomes.

\section{Normalization and Combining of Indices}

Normalization is required for bringing data with different ranges and units to a common level. This process is completed to enable further manipulation of the data and is rarely conducted for the mere purpose of normalization. However, normalization requires knowledge of the data and realization of the ultimate purpose of normalization. It is highly noticeable in both practice and research that regulations are leaning toward performancebased assessment; therefore, performance-based regulations are attracting attention [15]. Performance-based regulations were introduced in order to overcome several difficulties faced by customers. Utilities in the deregulated environment have one major objective: maximizing profit. Whether they accomplish this by minimizing loss, providing cheaper power, or poor quality power, regulators' roles in distribution systems become vital. Multiple methods are used for normalization: maximum, minimum, maximum norm, Euclidean norm, average, etc. These methods will normalize all indices mathematically but will not include in their normalization any known superiorities amongst systems. From an engineering perspective, equal indices in two systems do not necessarily reflect equal performance. Therefore, development of a new normalization methodology is necessary.

In [10], simple normalization to the maximum amongst load point indices will be sufficient to combine indices. Moreover, after normalization, weights are assigned by a reliability engineer in order to combine all indices. However, this is not equal in comparative studies and merely deals with the problem mathematically, without an under- 
standing of the problem. Another approach was made by [27]. In this work, some indices (reliability and power quality) were assigned weights ( $\mathrm{X} \$$ /unit index) in order to convert all indices into dollars; then, comparisons may be performed or further explored. However, this technique also suffers from equal basis as it normalizes by the maximum; this is assuming equal weights. In the case of different weights, comparative studies will become unfair because system reliability (service) performance should be made in similar environments to eliminate bias. For instance, an outage of a silicon factory will certainly not equal the value of an outage in an equally sized (loading) residential load. Thus, results will not directly reflect the performance of the design but will rather highlight how severe an outage is financially. Developing a completely new index which incorporates as many indices as possible was the methodology used in [29]. The author in [29] suggests a survey for distribution to customers in order to gain feedback on the question of effective time. Effective time was used as a compromise for what customers think of a specific outage duration. This methodology only reflects some indices. Moreover, it lacks the ability to aggregate the effects of system size and loading conditions. In other words, it is more reflective of reliability from customers' perspectives rather than service quality. Authors in [25] used a similar approach to [27]. However, in [25] the methodology involved reliability worth rather than assigning weights. This leads to the fundamental problem, as described in $[11,34,35]$, that using reliability worth in deciding which system is the better design is weak; therefore, they also reported that many utilities are adopting reliability indices based distribution system designs or performance-based assessment in the decisionmaking process. Analytic Hierarchy Process was used in [26] to unify indices. However, the authors did not include many of the recommended reliability indices by [20]. Moreover, the cost-based decision is eventually mimicked as cost dominates the decision.

\section{Problem Description}

Issues arise when trying to compare values of indices because of their conflicting nature $[25,26,29]$. If the frequency of interruption is low and the duration of the interruption of a load point is long, decisions can be challenging to make when compared with a load point with a higher frequency of interruption and shorter duration. Moreover, comparison and cross-comparison of reported data amongst utilities become inevitable for regulators [15]. However, the current infrastructure of indices does not promote fair and accurate comparison. Proceeding from the growing need to assess the performance of distribution systems in such a way that allows fair historical and current comparisons within one system (subsystems) and cross-comparisons between different systems and subsystems, this work tackles the goal of a simple, representative, and easily interpreted single index. The main objective of this single index is to evaluate distribution system performance using one number. The developed single number should be adequate for assessment and comparison purposes. Moreover, the derived index ought to reflect information from reliability indices. Therefore, a Unified Index based on all reliability indices recommended by the IEEE Guide for Electric Power Distribution Reliability Indices (IEEE Std 1366-2012) has been developed [20]. The developed Unified Index (UI) will accurately and fairly assess systems or subsystems without the need for highly qualified personnel. In addition, the UI will carry information from all indices and will reflect major components of systems topology in terms of customer count, loading level, and a number of serving points (i.e. load points). This UI will also allow for penalty/reward policies to be implemented. The methodology and steps toward reaching the UI are elaborated. The selected indices must reflect the entire system performance with regard to optimization. This means that if these indices were to be optimized, the best possible system performance would be achieved. Then, the normalization part of the problem is presented and modeled. The normalized numbers should reach a place that overcomes some of the aforementioned difficulties in cross-comparisons and comparative studies. Proceeding from the selected normalized indices, the combination phase illustrates the methodology used in order to combine all different indices into one UI reflective of the overall system performance in terms of reliability.

\section{Problem Formulation}

The following Eq. 1 represents a general formulation for a multi-objective UI. This general formulation can be used in a variety of studies, except comparative ones. In addition, the weights are unknown and need to be assigned. However, there is not one precise methodology for assigning values to these weights. By using the general formulation, some algebraic manipulations are made to reflect the correlation between indices and system size effect.

$$
\begin{aligned}
\mathrm{UI}=w_{1} \frac{\mathrm{SAIFI}_{\text {actual }}}{\mathrm{SAIFI}_{\text {base }}}+w_{2} \frac{\mathrm{SAIDI}_{\text {actual }}}{\mathrm{SAIDI}_{\text {base }}} \\
+w_{3} \frac{\mathrm{CAIFI}_{\text {actual }}}{\mathrm{CAIFI}_{\text {base }}}+w_{4} \frac{\mathrm{CAIDI}_{\text {actual }}}{\mathrm{CAIDI}_{\text {base }}} \\
+w_{5} \frac{\mathrm{ASIFI}_{\text {actual }}}{\mathrm{ASIFI}_{\text {base }}}+w_{6} \frac{\mathrm{ASIDI}_{\text {actual }}}{\mathrm{ASIDI}_{\text {base }}} \\
+w_{7} \frac{\text { MAIFI }_{\text {actual }}}{\text { MAIFI }_{\text {base }}}+w_{8} \frac{\text { MAIFI }_{\mathrm{E}_{\text {actual }}}}{\mathrm{MAIFI}_{\mathrm{E}_{\text {base }}}}
\end{aligned}
$$




$$
\begin{aligned}
& +w_{9} \frac{\mathrm{CTAIDI}_{\text {actual }}}{\mathrm{CTAIDI}_{\text {base }}}+w_{10} \frac{\mathrm{ASAI}_{\text {actual }}}{\mathrm{ASAI}_{\text {base }}} \\
& +w_{11} \frac{\mathrm{CEMI}_{\mathrm{n}_{\text {actual }}}}{\mathrm{CEMI}_{\mathrm{n}_{\text {base }}}}+w_{12} \frac{\mathrm{CEMSMI}_{\mathrm{n}_{\text {actual }}}}{\mathrm{CEMSMI}_{\mathrm{n}_{\text {base }}}} \\
& +w_{13} \frac{\mathrm{CELID- \textrm {t } _ { \text { actual } }}}{\mathrm{CELID}_{\mathrm{t}_{\text {base }}}}+w_{14} \frac{\mathrm{CELID- \textrm {s } _ { \text { actual } }}}{{\mathrm{CELID}-\mathrm{s}_{\text {base }}}^{\text {CEL }}}
\end{aligned}
$$

Assuming that the two indices $\mathrm{CEMI}_{\mathrm{n}}$ and $\mathrm{CEMSMI}_{\mathrm{m}}$ are being calculated for a specific value of $n$ (number of sustained interruption) and $\mathrm{m}$ (number of sustained and momentary interruptions), expanding and rearranging the general equation;

After further simplification and rearrangement, the final equation is reached.

For $\mathrm{CEMI}_{\mathrm{k}_{\text {base }}}$ and $\mathrm{CEMSMI}_{\mathrm{l}_{\text {base }}}$, worst-case scenarios are when both equal to one. Therefore, the weights will be; $\mathrm{CEMI}_{\mathrm{k}_{\text {base }}}=\mathrm{CEMSMI}_{\mathrm{l}_{\text {base }}}=1$ For $\mathrm{CAIDI}_{\text {base }}$, values of $\mathrm{SAIFI}_{\text {base }}$ and $\mathrm{SAIDI}_{\text {base }}$ can be used instead. By doing so we arrive to; $\mathrm{CAIDI}_{\text {base }}=\mathrm{SAIDI}_{\text {base }} / \mathrm{SAIFI}_{\text {base }}$ For $\mathrm{CAIFI}_{\text {base }}$ and $\mathrm{CTAIDI}_{\text {base, }}$, worst-case scenarios for both indices are being equal to $\mathrm{SAIFI}_{\text {base }}$ and $\mathrm{SAIDI}$ base respectively. Because the denominator of both $\mathrm{CAIFI}_{\text {base }}$ and $\mathrm{CTAIDI}_{\text {base}}$, in the worst-case scenario, will be equal to the total number of customers served, yielding values equal to SAIFI base and SAIDI base. This is true with the fact that the nominators of CAIFI base $_{\text {and }}$ SAIFI base are always equal and nominators of CTAIDI ${ }_{\text {base }}$ and $\mathrm{SAIDI}_{\text {base }}$ are also always equal. This will yield to; $\mathrm{CAIFI}_{\text {base }}=\mathrm{SAIFI}_{\text {base }}$ and $\mathrm{CTAIDI}_{\text {base }}=\mathrm{SAIDI}_{\text {base }}$

For ASUI $_{\text {base, }}$, it can be noticed that ASUI $=$ SAIDI $/ 8760$. The number (8760) represents the total number of hours in a year. This number can be changed according to the common usage of hours in a year. However, it is irrelevant in this specific case, as the same number will eventually be multiplied by the $\mathrm{ASUI}_{\text {base }}$ again. Therefore; $\mathrm{ASUI}_{\text {base }}=$ $\mathrm{SAIDI}_{\text {base }} / 8760$

Finally, for $\mathrm{ASIFI}_{\text {base }}$ and ASIDI $\mathrm{A}_{\text {base }}$, these indices differ from $\mathrm{SAIFI}_{\text {base }}$ and $\mathrm{SAIDI}_{\text {base }}$ in non-homogeneous systems only. The definition of homogeneous used here is that the ratio of the total number of customers served and the total KVA or KW of the system is 1. Therefore, one base can be used for both under the condition of being the largest. By choosing a SAIFI ${ }_{\text {base }}$ and SAIDI base larger than ASIFI and ASIDI, which is common as the values of SAIFI and SAIDI are usually larger than ASIFI and ASIDI, we will reach; $\mathrm{ASIFI}_{\text {base }}=\mathrm{SAIFI}_{\text {base }}$ and $\mathrm{ASIDI}_{\text {base }}$ $=\mathrm{SAIDI}_{\text {base }}$

For $\mathrm{SAIFI}_{\text {base }}$, in general, if normalizing to the maximum, it should not be less the maximum frequency of interruption within the components of the system under study. Therefore; the equation can be written as follows in Eq. 2 .

$$
\begin{aligned}
\mathrm{UI} & =\frac{\frac{w_{1} \mathrm{CI}}{\mathrm{N}_{\mathrm{T}}}+\frac{w_{3} \mathrm{CI}}{\mathrm{CN}}}{\mathrm{SAIFI}}+\frac{\frac{w_{7} \mathrm{MAIFI}_{\text {actual }}+w_{8} \mathrm{MAIFI}_{\mathrm{E}_{\text {actual }}}}{\mathrm{N}_{\mathrm{T}}}}{\mathrm{MAIFI}_{\text {base }}} \\
& +\frac{w_{11} \mathrm{CN}_{\mathrm{k}>\mathrm{n}}+w_{12} \mathrm{CNT}_{\mathrm{k}>\mathrm{n}}+w_{13} \mathrm{CN}_{\mathrm{k}>\mathrm{T}}+w_{14} \mathrm{CN}_{\mathrm{k}>\mathrm{S}}}{\mathrm{N}_{\mathrm{T}}} \\
& +\frac{\frac{w_{9} \mathrm{CMI}}{\mathrm{CN}}+\frac{\left(w_{2}+w_{10}\right) \mathrm{CMI}}{\mathrm{N}_{\mathrm{T}}}+\frac{w_{4} \mathrm{CMI} \mathrm{SAIFI}_{\text {base }}}{\mathrm{CI}}}{\mathrm{SAIDI}_{\text {base }}}
\end{aligned}
$$

\section{The Proposed Methodology}

\subsection{Normalization}

The previously mentioned methods for normalization in the literature are broadly used. However, normalizing by maximum, minimum, norm, or any other method of normalization that uses self-data, is not sufficient. Therefore, for the purpose of achieving the objectives of this work, we assume that they carry the same problems. For the purpose of reaching a unified reliability index, a new normalization technique is developed. In this technique, the problem of having indices with different ranges and weights is overcome. In addition, the normalized indices will be comparison-ready after normalization. The key idea in this normalization methodology is using more information to distinguish between one system and the other. For instance, two systems with the same final SAIFI values do not necessarily report equal performance in a distribution engineering sense. They provide a mere number of how many times an average customer of this system has been interrupted during the study period. However, one of the systems could be significantly larger than the other. Thus, the larger system is more susceptible to outages and events. In the engineering sense of the results, the larger system should reflect some better performance indications compared to the smaller system. Though the current indices are calculated based on an average customer or average unit of power basis, it is unfair to compare a whole system with a relatively large number of customers and an excellent loading level with one that has a smaller number of customers and lower loading levels. Therefore, the normalization will be conducted similarly to the per unit system in power systems. In the per unit system, the values are calculated based on a base value that has been assigned or calculated from other bases. Similarly, the base values of each system will be different from the others (3). For example, in a power system, the voltage base in a line can be different from the voltage base in the bus or the generator. Consequently, bases for each system will be calculated according to the same idea. Some bases will be assigned, and others will be calculated.

$\mathrm{SAIFI}_{\text {base }}=\frac{\lambda_{s \max _{(l)}}+\lambda_{s \max _{(g)}}}{2}$ 
$\mathrm{SAIDI}_{\text {base }}=\frac{\left(\lambda_{s} \times r_{s}\right)_{\max _{(l)}}+\left(\lambda_{s} \times r_{s}\right)_{\max _{(g)}}}{2}$

MAIFI $_{\text {base }}=\frac{\lambda_{m \max _{(l)}}+\lambda_{m \max _{(g)}}}{2}$

\subsection{Unification}

Weights play a significant role in deciding which system is performing best. In any case, all weights should be kept constant among all systems under study, and their summation must be equal to one. The common ways for assigning these values are either by experience or relative cost (reliability worth) of each index.

In this section, the problem of assigning individual weights for each index of the IEEE Std 1366-2012 [20] is tackled. These weights play a significant role in deciding which system is performing best. In any case, all weights should be kept constant among all systems under study, and their summation must be equal to one. The common ways for assigning these values are either by experience or relative cost (reliability worth) of each index.

Three main methodologies are presented in this paper to combine the indices namely, simple averaging (equal weights), different weights, and fuzzy inference based. The development of different weights require experience and differ from one regulator to another; thus, in this work, development of an optimization problem to find the weights that yield minimum performance index globally has been conducted. The other two techniques were used for verification by comparing their perforce with the new normalization and new weights scheme. The following subsections discuss these methods and propose the new method for this task.

\subsubsection{Equal-Weight Unification}

One simple way to combine theses indices is to give each an equal weight (i.e. averaging). This approach is not practical, as indices differ in their impact on reliability, so it is beneficial to perform quick assessments, especially when weights are unknown and the systems under study have a similar topology. In such cases, the effect of each index toward the UI is the same.

\subsubsection{Different-Weight Unification}

Depending on the impact each index has, different weights can be assigned to each index. The difference in weights comes from many factors. For instance, an industry type that is concerned with the duration of each interruption, rather than how many short interruptions happen, should be assigned larger weights from the duration indices. Other industries may reflect dissatisfaction with the frequency of interruptions regardless of duration, and these must be assigned different weights. A general consensus regarding this issue is hard to achieve. In a general sense, these weights depend on the authority performing such studies and will differ between one authority and another. Alternatively, an optimization problem needs to be solved to evaluate the weights. The optimization problem minimizes the sum of all unified indices of all systems by finding the optimal individual weights. Equation 6 can be used as an objective function. In doing so, the control variables are the individual weights, leading to a minimum sum. However, in reliability-based system planning, the objective is usually the associated cost i.e., reliability worth. This reliability-enhancing objective is irrelevant in assessments and comparisons especially in cross-comparisons due to the difference in system topologies. To avoid neglecting some weights, minimum and maximum values are used as constraints as stated in Equations 7, 8, and 9.

Obj: $\min \sum_{i \in \mathscr{I}} \mathrm{UI}_{i}$

where:

$$
\begin{aligned}
\sum_{x \in \mathscr{X}} w_{x} & =1 \\
\mathrm{UI}_{i} & \leq 1 \\
\mathrm{LB} & \leq w_{x} \leq \mathrm{UB}
\end{aligned}
$$

\subsubsection{Fuzzy Inference}

Fuzzy sets and fuzzy logic were first discussed in 1965 by Zadeh [37]. They have acquired great attention from researchers since their development. Many uses and implementation have been made using them. Their robustness and simplicity by using linguistic interpretations of phenomenon escalated their adaptation. Further theorems and developments have been added after their first light [23]. In the proposed methodology, a fuzzy inference knowledge-based engine is developed. Similar to ideas developed by $[4,19$, 22,32] for relative importance between inputs, a knowledgebased fuzzy inference system was developed. With 14 inputs (reliability indices) and one output (ranking criterion), decisions are made based on knowledge-based rules [24]. These rules are made using a program. The program follows a creation criterion that checks the state of each input and allows for proper consequence. It assigns values of the membership for each index level. For instance, Table 1 provides an example of some of the IF-THEN rules acquired by the program.

The MATLAB Fuzzy Logic Toolbox was utilized to create the fuzzy inference functions. The fuzzy inference functions used in this approach are as follows: 
Table 1 Example of developed rules

\begin{tabular}{llll}
\hline IF & & THEN \\
\hline SAIFI & Low & System & Very reliable \\
SAIDI & Low & \\
CAIFI & Low & \\
CAIDI & Low & \\
ASIFI & Low & \\
ASIDI & Low & \\
CTAIDI & Low & \\
ASUI & Low & \\
MAIFI & Low & \\
MAIFIe & Low & \\
CEMI & Low & \\
CEMSMI & Low & \\
CELID-t & Low & \\
CELID-s & Low & \\
\hline
\end{tabular}

- Name $=$ 'Fuzzyinf2'

- Type $=$ 'mamdani'

- Version $=2.0$

- NumInputs $=14 ;$ NumOutputs $=1 ;$ NumRules $=19683$

- AndMethod = 'min'; OrMethod = 'max'; ImpMethod $=$ 'min'

- AggMethod = 'max'; DefuzzMethod = 'centroid'

- An example of input fuzzification functions is as follows:

- Name $=$ 'SAIFI'

- Range $=\left[\begin{array}{ll}0 & 1\end{array}\right]$

- NumMFs $=3$

- MF1 = 'Low': 'trimf', $\left[\begin{array}{lll}-0.5 & 0 & 0.5\end{array}\right]$

- MF2 = 'Medium': 'trimf', [0 0.51$]$

- MF3 = 'High':'trimf', [0.5 11.5$]$

In Figs. 1 and 2, an example of fuzzy membership functions of an input and the membership functions of the output is illustrated. These functions were developed according to prior knowledge of system reliability performance expectations. Further, by definition, all reliability indices used in this work follow similar behaviour i.e., the lower the value, the higher the reliability.

\section{Verification and Testing}

In [2], the two test systems provided are, first, a 38-load-point with seven feeders system (i.e. Bus 4 in Fig. 4a) and, second, a 22-load-point 4-feeder system (i.e. Bus 2 in Fig. 4b). The reference provides comprehensive data on the two systems with regard to loading and failure rates for two cases: lines or cables. Moreover, the paper suggests different (six for every case) protection and restoration topologies. As a result, the

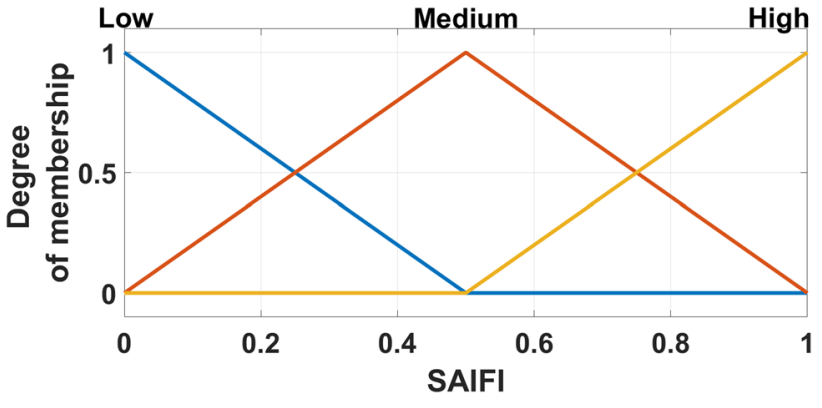

Fig. 1 SAIFI membership function

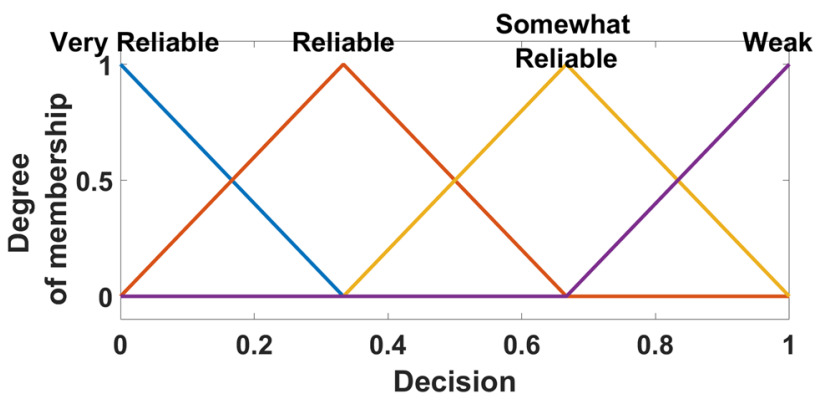

Fig. 2 Output membership function

two systems with the two cases of lines or cables and the six different topologies yield to a total number of options for each test system of 12. In this section, the methodology described in this work will be implemented and compared with the explicit and implicit ranking described in [2]. The aforementioned test systems have several topologies. The values are either directly quoted from [2] or calculated (synthesized) with accordance to [20]. In these test systems, the different weights' optimization problem yielded the values listed in Table 5. GAMS package was used and the solver for this problem was MINOS. The solver is designed to solve linear and nonlinear programming problems by finding a local optimal. The linear constraints define an area in a problem and by the bounds on the variables. Convexity of the objective and constraints in the defined region guarantees that the found local optimal is indeed a global one. Linearly constrained models are then solved with a gradient technique that takes advantage of the sparsity of the model. For models with nonlinear constraints and objective, iterative relaxation (linearization and Lagrangian objective) of sub-problems are solved. These techniques are commonly used for economic dispatch problems in power systems (Fig. 3).

\subsection{Example of a Typical Power System Optimization}

An example and its solution of an economic dispatch problem is described in Fig. 3. The mathematical modelling is performed in Eqs. 10-18. Parameters of system, the costs of 
Fig. 3 Results of the example power system economic dispatch problem

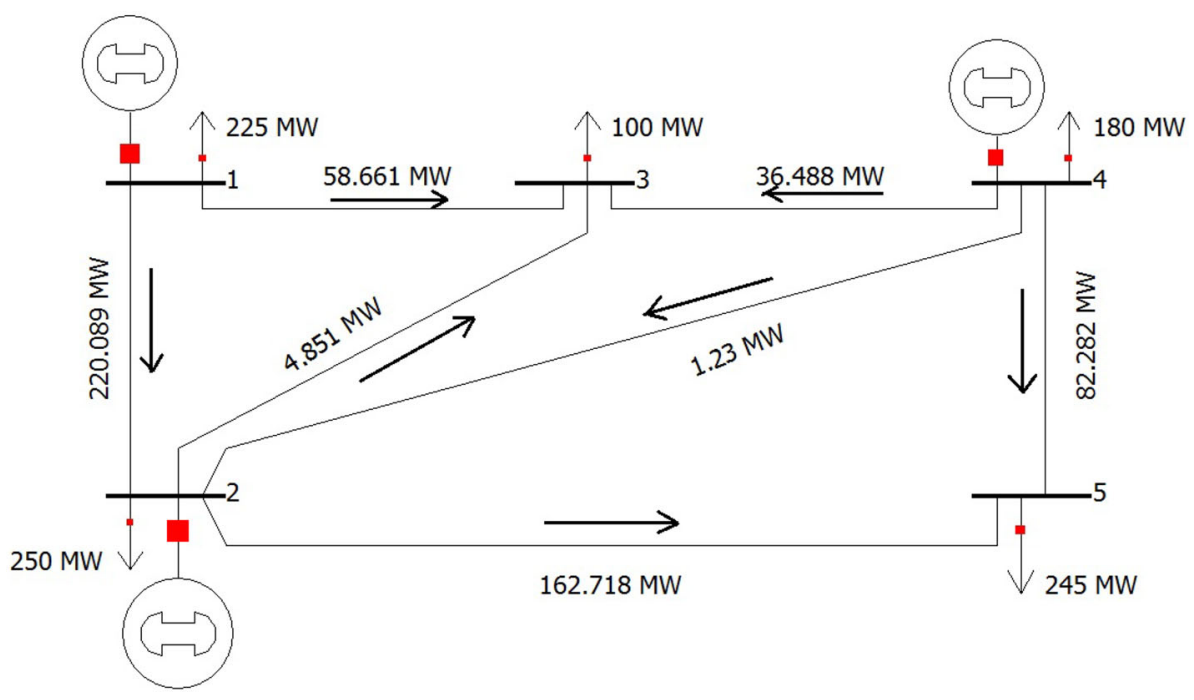

Fig. 4 Test systems [2]. a Bus 4 , b Bus 2

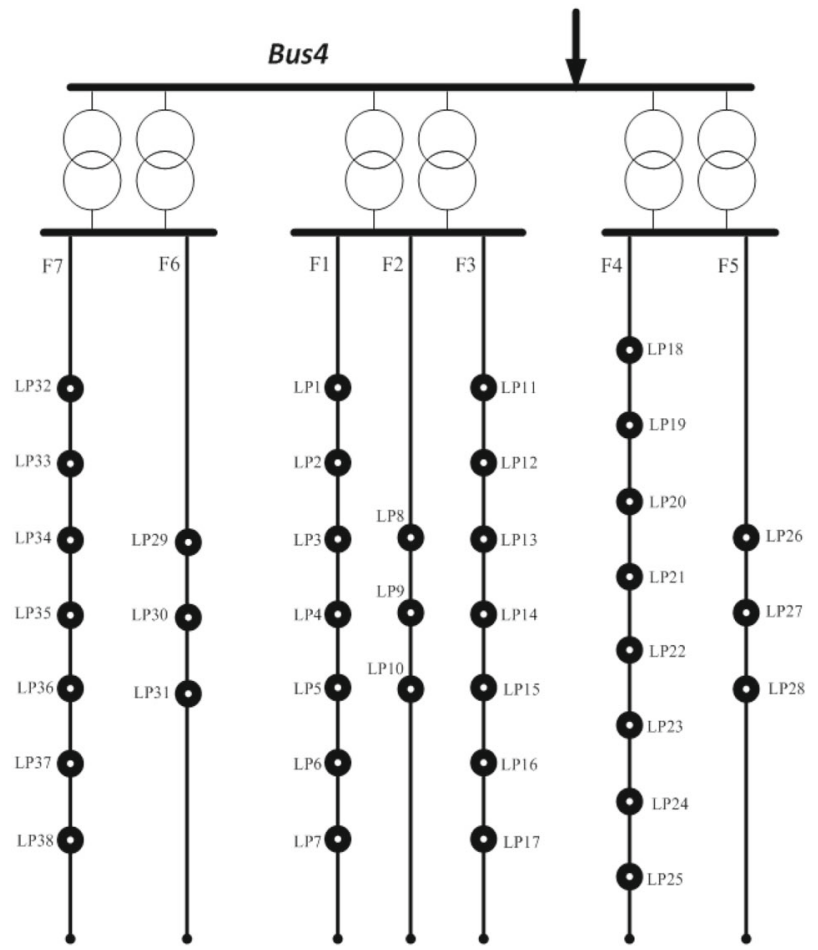

(a)

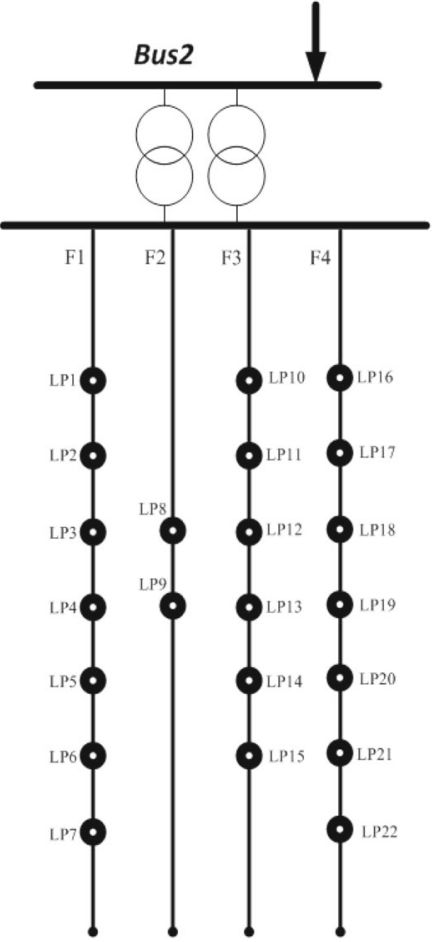

(b) generation, and loading are summarized in Tables 2, 3, and 4. The standard cost function is given by:

$C_{i}=a_{i} P_{i}^{2}+b_{i} P_{i}+c_{i}$,

and the objective function is:

$\min J=\sum_{i \in I} C_{i}\left(P_{i}\right)$, subject to loading/generation balance:

$1000-\left(P_{g 1}+P_{g 2}+P_{g 4}\right)=0$,

and also subject to generation limits:

$$
\begin{aligned}
& P_{g 1}-600 \leq 0 \\
& P_{g 2}-400 \leq 0 \\
& P_{g 4}-300 \leq 0
\end{aligned}
$$


Table 2 Generators cost coefficients

Table 3 Buses' real power demands

Table 4 Line parameters

$$
\begin{array}{r}
150-P_{g 1} \leq 0 \\
100-P_{g 2} \leq 0 \\
50-P_{g 4} \leq 0 .
\end{array}
$$

This convex formulation can be extended to include power flow equations described in (19) and (20) for active and reactive power. These equations impose nonconvexity due to their nonlinear nature (Table 5).

$P_{g_{i}}-P_{D i}=\sum_{j \in \mathscr{J}} V_{i} V_{j} Y_{(i, j)} \cos \left(\theta_{(i, j)}+\delta_{j}-\delta_{i}\right) \forall i \in \mathscr{I}$

$Q_{g_{i}}-Q_{D i}=-\sum_{j \in \mathscr{J}} V_{i} V_{j} Y_{(i, j)} \sin \left(\theta_{(i, j)}+\delta_{j}-\delta_{i}\right) \forall i \in \mathscr{I}$

where

$i, j$ and $\mathscr{I}, \mathscr{J}$ : bus indices and system bus sets respectively;
Table 5 Weights obtained from optimization

\begin{tabular}{lllllll}
\hline$w_{1}$ & $w_{2}$ & $w_{3}$ & $w_{4}$ & $w_{5}$ & $w_{6}$ & $w_{7}$ \\
\hline 0.0286 & 0.0286 & 0.1143 & 0.1143 & 0.0286 & 0.0286 & 0.1143 \\
$w_{8}$ & $w_{9}$ & $w_{10}$ & $w_{11}$ & $w_{12}$ & $w_{13}$ & $w_{14}$ \\
0.1143 & 0.0286 & 0.1143 & 0.0286 & 0.0286 & 0.1143 & 0.1143 \\
\hline
\end{tabular}

\subsection{Optimization Results of the Proposed Technique}

In Table 6, results of the ranking compared with simple normalization and averaging technique is illustrated. It can be noticed how the proposed methodology reflects the superiority of larger systems with similar indices but differ in topology and size.

The goal of this section is to verify whether or not the known assessments and ranks are achieved. In order to do so, case studies will be presented and studied. These cases have one thing in common: rank is known. First, systems which are relatively similar, and with known rank, are studied. Second, systems with relatively different topologies but approximately equal indices are analyzed. In [2], the two test systems provided are, first, a 38-load-point with 7 feeders system (i.e. Bus4 in Fig. 1) and, second, a 22-load-point 4-feeder system (i.e. Bus2 in Fig. 2). The reference provides comprehensive data on the two systems with regard to loading and failure rates for two cases: lines or cables. Moreover, the paper suggests different (six for every case) protection and restoration topologies. As a result, the two systems with the two cases of lines or cables and the six different topologies yield to a total number of options for each test system of 12. In this section, the methodology described in this work will be implemented and compared with the explicit and implicit ranking described in [2]. The aforementioned test systems have several topologies; these will be coded and described in the Table 7 in Appendix. The values are either directly quoted from [2] or calculated with accordance to [20]. In these test systems, the different weights optimization problem yielded the values listed in Table 2. Values are compared with the equal weights method to illustrate the difference.

In Table 6, the ranking is presented with regard to reliability performance. A major advantage of the fuzzy approach, which can be seen in the results, is the relative importance of different reliability indices. The proposed simpler methodology is consitant with the fuzzy inference ranking. Decisions reached by an engine with vast number of rules was also reached using the proposed normalization and unification techniques. The ranking here indicates better reliability performance (i.e., smaller UI).

Figure 5 illustrates the output results of the scaled average (equal weights) and the fuzzy approach. It can be noted that 
Table 6 Ranking results of fuzzy approach

\begin{tabular}{|c|c|c|c|c|c|c|}
\hline & \multicolumn{3}{|l|}{ Results } & \multicolumn{3}{|l|}{ Rank } \\
\hline & Simple & Proposed & Fuzzy & Simple & Proposed & Fuzzy \\
\hline AL4 & 0.157232 & 0.225714 & 0.3853 & EL2 & EL4 & EL4 \\
\hline BL4 & 0.702827 & 0.769369 & 0.5182 & EL4 & EL2 & EL2 \\
\hline CL4 & 0.186092 & 0.26493 & 0.4436 & $\mathrm{EC} 4$ & $\mathrm{EC} 4$ & $\mathrm{EC} 4$ \\
\hline DL4 & 0.247323 & 0.315862 & 0.4477 & $\mathrm{EC} 2$ & $\mathrm{EC} 2$ & $\mathrm{EC} 2$ \\
\hline EL4 & 0.070831 & 0.097318 & 0.2111 & AL4 & AL4 & AL4 \\
\hline FL4 & 0.413602 & 0.513306 & 0.5081 & AL2 & AL2 & AL2 \\
\hline $\mathrm{AC} 4$ & 0.19425 & 0.275167 & 0.4448 & CL4 & CL4 & CL4 \\
\hline $\mathrm{BC} 4$ & 0.942737 & 0.949312 & 0.6744 & CL2 & CL2 & CL2 \\
\hline $\mathrm{CC} 4$ & 0.382505 & 0.491841 & 0.5039 & $\mathrm{AC} 4$ & $\mathrm{AC} 4$ & $\mathrm{AC} 4$ \\
\hline DC4 & 0.265804 & 0.360041 & 0.4647 & $\mathrm{AC} 2$ & DL4 & DL4 \\
\hline EC4 & 0.087373 & 0.12953 & 0.3544 & DL4 & $\mathrm{AC} 2$ & $\mathrm{AC} 2$ \\
\hline FC4 & 0.527815 & 0.635934 & 0.5155 & DC4 & DC4 & DC4 \\
\hline AL2 & 0.168259 & 0.243 & 0.4114 & DL2 & DL2 & DL2 \\
\hline BL2 & 0.702827 & 0.769369 & 0.5567 & $\mathrm{DC} 2$ & $\mathrm{FC} 2$ & $\mathrm{FC} 2$ \\
\hline CL2 & 0.187197 & 0.268288 & 0.4446 & $\mathrm{FC} 2$ & $\mathrm{CC} 2$ & $\mathrm{CC} 2$ \\
\hline DL2 & 0.282312 & 0.370401 & 0.4753 & $\mathrm{CC} 2$ & $\mathrm{DC} 2$ & DC2 \\
\hline EL2 & 0.070445 & 0.101184 & 0.2575 & FL2 & FL2 & FL2 \\
\hline FL2 & 0.365357 & 0.469651 & 0.4994 & $\mathrm{CC} 4$ & $\mathrm{CC} 4$ & $\mathrm{CC} 4$ \\
\hline $\mathrm{AC} 2$ & 0.242596 & 0.33371 & 0.457 & FL4 & FL4 & FL4 \\
\hline $\mathrm{BC} 2$ & 0.92281 & 0.95122 & 0.6749 & $\mathrm{FC} 4$ & $\mathrm{FC} 4$ & FC4 \\
\hline $\mathrm{CC} 2$ & 0.340154 & 0.444992 & 0.4888 & BL4 & BL4 & BL4 \\
\hline $\mathrm{DC} 2$ & 0.335119 & 0.446702 & 0.495 & BL2 & BL2 & BL2 \\
\hline $\mathrm{EC} 2$ & 0.120878 & 0.177374 & 0.3806 & $\mathrm{BC} 2$ & $\mathrm{BC} 4$ & $\mathrm{BC} 4$ \\
\hline $\mathrm{FC} 2$ & 0.338672 & 0.441167 & 0.4776 & $\mathrm{BC} 4$ & $\mathrm{BC} 2$ & $\mathrm{BC} 2$ \\
\hline
\end{tabular}

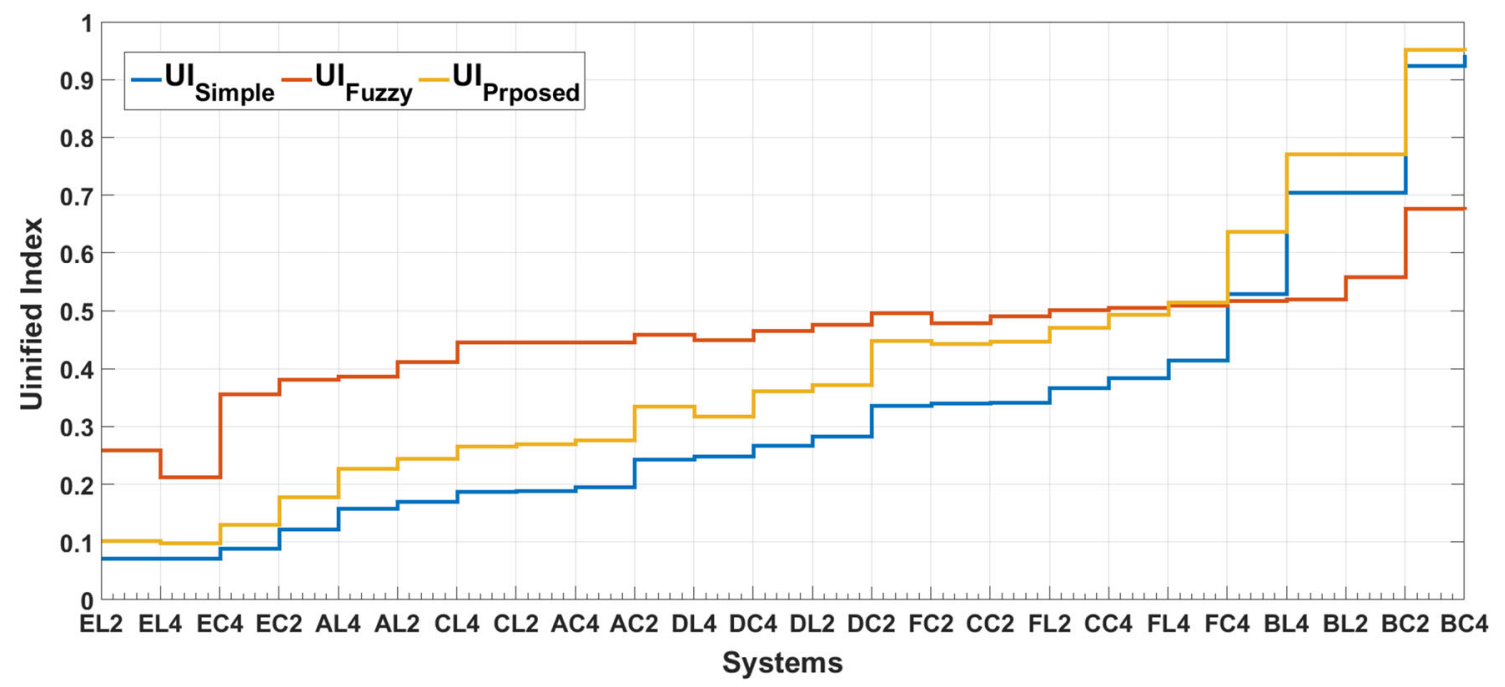

Fig. 5 Sorted results of simple, fuzzy and proposed unified indices

the scaled average methodology results in a relatively greater distances between values compared to the fuzzy approach. It can also be noticed the effect of relative importance in the fuzzy approach in what is considered high or low in scaled average methodology. The smoother output in the fuzzy approach allow for more reliable ranking decisions. 
It can be noticed that with the proposed methodology, much less technically challenging than fuzzy-based technique, is performing as well as the fuzzy system.

\section{Conclusion}

Reliability studies, though not new, are becoming increasingly important under the new framework and systems structure. Smart grids require advanced tools to assess and reflect adequate systems performance. The knowledge about distribution systems behavior and patterns is immense. However, this knowledge has not been fully adapted into knowledge-based performance assessment. Approximate reasoning and conceptual representations point to a very promising approach for performance analysis in distribution systems reliability. Utilities are coming under greater pressure as they strive to enhance their reliability while regulators cannot fully reflect on current reported numbers. Because of different systems topologies, comparing or perceiving reliability indices of two different systems can be misleading.

The fuzzy inference has shown robust decision-making capabilities with regard to systems reliability performance. It does not require normalization and can be modified to incorporate any particular needs in assessments. This work can be used to assess systems and subsystems such as feeders or load points. However, it is not a simple task to continuously develop this large number of rules.

In this work, a novel normalization methodology has been developed. The new methodology does not require customer surveys or customer interaction. This is beneficial as service quality, from a utility perspective, should remain unbiased and independent of customer type. Using the developed nor- malization methodology, the single index comparison is more reliable. As reporting data is routinely practiced by utilities, no major infrastructure or regulatory changes are required. A single index will have a different ranking on multiple systems; thus, that index's impact varies from system to system. The optimization-like problem was formed to decide the best weights to use across systems; then, indices combined to form a unified reliability index were implemented. Mathematical manipulation was conducted to relax and ease the general formulation of the unified reliability index. This yields a relaxed reporting routine. The UI was compared with the performance of the most practical and ready methodology in order to compare and cross-compare systems among each other and from the previous reporting period. In conclusion, steps toward a comprehensive reliability UI have been made. The results showed a unified reliability index capable of fair and accurate utility quality assessment. Though all reliability indices provide important information, they can be further analyzed for feature extraction and selection in the future. Further analysis is needed on using the number obtained in planning and operational optimization.

Acknowledgments The first author would like to acknowledge King Abdulaziz University for sponsoring his $\mathrm{PhD}$ studies and research.

\section{Appendix}

See Table 7 


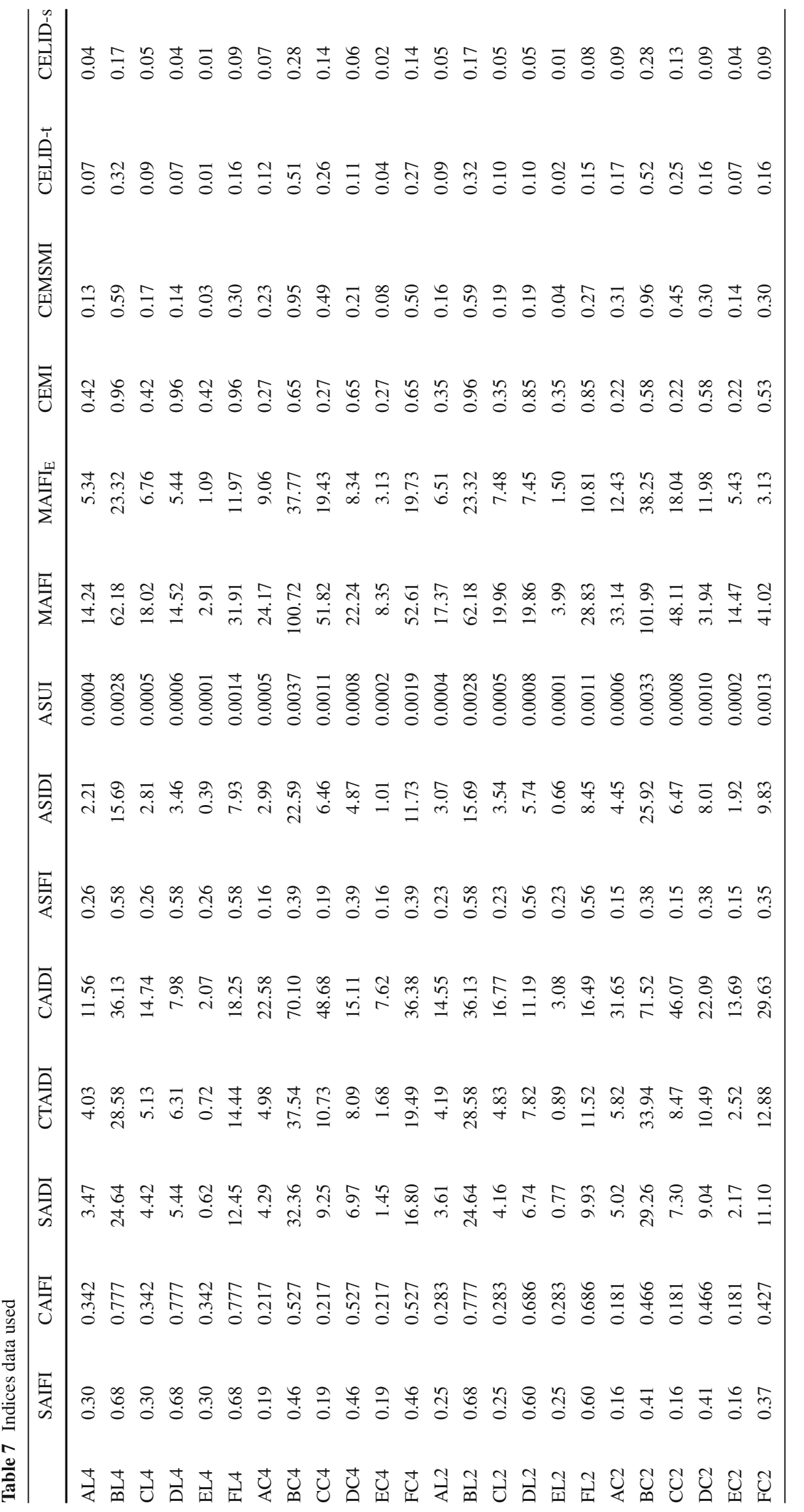




\section{References}

1. Abbasi, E., Fotuhi-Firuzabad, M., Abiri-Jahromi, A.: Risk based maintenance optimization of overhead distribution networks utilizing priority based dynamic programming. In: 2009 IEEE Power \& Energy Society General Meeting, pp. 1-11 (2009). doi:10.1109/ PES.2009.5275735

2. Allan, R.N., Billinton, R., Sjarief, I., Goel, L., So, K.S.: A reliability test system for educational purposes-basic distribution system data and results. IEEE Trans. Power Syst. 6(2), 813-820 (1991). doi:10. $1109 / 59.76730$

3. Alvehag, K., Soder, L.: Risk-based method for distribution system reliability investment decisions under performance-based regulation. IET Gener. Transm. Distrib. 5(10), 1062 (2011). doi:10.1049/ iet-gtd.2011.0047

4. Androshchuk, A.S.: Risk assessment of offences at state border using fuzzy hierarchical inference. Theory Appl. p. 72

5. Balijepalli, N., Venkata, S.S., Christie, R.D.: Modeling and analysis of distribution reliability indices. IEEE Trans. Power Deliv. 19(4), 1950-1955 (2004). doi:10.1109/TPWRD.2004.829144

6. Billinton, R.: Distribution system reliability performance and evaluation. Int. J. Electr. Power Energy Syst. 10(3), 190-200 (1988)

7. Billinton, R., Bollinger, K.E.: Transmission system reliability evaluation using Markov processes. IEEE Trans. Power Appar. Syst. PAS-87(2), 538-547 (1968). doi:10.1109/TPAS.1968.292051

8. Billinton, R., Allan, R.N.: Reliability evaluation of power systems, vol. 2. Plenum Press, New York (1984)

9. Brown, R.E.: Distribution reliability assessment and reconfiguration optimization. In: 2001 IEEE/PES Transmission and Distribution Conference and Exposition, vol. 2, pp. 994-999 (2001). doi:10. 1109/TDC.2001.971382

10. Brown, R.E.: Electric Power Distribution Reliability. CRC Press, Boca Raton (2008)

11. Brown, R.E., Spare, J.H.: A survey of reliability reporting practices. In: 2005/2006 IEEE PES Transmission and Distribution Conference and Exhibition, pp. 1212-1217 (2006)

12. Brown, H.E., Suryanarayanan, S.: A survey seeking a definition of a smart distribution system. In: 41st North American Power Symposium, vol. 80401, pp. 1-7 (2009). doi:10.1109/NAPS.2009. 5484078

13. Brown, R.E., Gupta, S., Christie, R.D., Venkata, S.S., Fletcher, R.: Distribution system reliability assessment using hierarchical Markov modeling. IEEE Trans. Power Deliv. 11(4), 1929-1934 (1996). doi:10.1109/61.544278

14. Brown, R.E., Gupta, S., Christie, R.D., Venkata, S.S., Fletcher, R.: Distribution system reliability assessment: momentary interruptions and storms. IEEE Trans. Power Deliv. 12(4), 1569-1575 (1997). doi:10.1109/61.634177

15. Chowdhury, A., Koval, D.: Power Distribution System Reliability: Practical Methods and Applications, vol. 48. Wiley, New York (2011)

16. Fraser, J.: Annual Reporting of Reliability Indices (Annual Response to Directive 26 of BCUC Decision on F05/F06 RRA). Tech. rep, BC hydro Annual Reporting (2012)

17. Fumagalli, E., Black, J.W., Vogelsang, I., Ilic, M.: Quality of service provision in electric power distribution systems through reliability insurance. IEEE Trans. Power Syst. 19(3), 1286-1293 (2004). doi:10.1109/TPWRS.2004.831294

18. Gaver, D.P., Montmeat, F.E., Patton, A.D.: Power system reliability I-measures of reliability and methods of calculation. IEEE Trans. Power Appar. Syst. 83(7), 727-737 (1964). doi:10.1109/ TPAS.1964.4766068

19. Guimarães, A.C.F., Lapa, C.M.F.: Fuzzy inference to risk assessment on nuclear engineering systems. Appl. Soft Comput. 7(1), $17-28$ (2007)
20. IEEE Guide for Electric Power Distribution Reliability Indices. IEEE Std 1366-2012 (Revision of IEEE Std 1366-2003), pp. 1-43 (2012). doi:10.1109/IEEESTD.2012.6209381

21. IEEE Recommended Practice for the Design of Reliable Industrial and Commercial Power Systems (IEEE Gold Book). ANSI/IEEE Std 493-1980, pp. 1-216 (2013). doi:10.1109/IEEESTD.2013. 6413301

22. Iranmanesh, S.H., Khodadadi, S.B., Taheri, S.: Risk Assessment of Software Projects Using Fuzzy Inference System. In: International Conference on Computers \& Industrial Engineering (CIE), pp. 1149-1154 (2009)

23. Klir, G., Yuan, B.: Fuzzy Sets and Fuzzy Logic, vol. 4. Prentice Hall, Upper Saddle River (1995)

24. Lang, B.P., Pahwa, A.: Power distribution system reliability planning using a fuzzy knowledge-based approach. IEEE Trans. Power Deliv. 15(1), 279-284 (2000)

25. Lee, B., Kim, K.M.: Unified power quality index based on valuebased methodology. In: IEEE Power \& Energy Society General Meeting, pp. 1-8 (2009). doi:10.1109/PES.2009.5275471

26. Lee, B., Kim, K.M., Goh, Y.: Unified power quality index using ideal AHP. In: 2008 13th International Conference on Harmonics and Quality of Power, pp. 1-5 (2008). doi:10.1109/ICHQP.2008. 4668807

27. McGranaghan, M.F.: Quantifying reliability and service quality for distribution systems. IEEE Trans. Ind. Appl. 43(1), 188-195 (2007). doi:10.1109/TIA.2006.886990

28. Montmeat, F.E., Patton, A.D., Zemkoski, J., Cumming, D.J.: Power system reliability II-applications and a computer program. IEEE Trans. Power Appar. Syst. 84(7), 636-643 (1965). doi:10.1109/ TPAS.1965.4766235

29. Ortmeyer, T.: A unified index for power distribution reliability/power quality indices. In: Proceedings of 14th International Conference on Harmonics and Quality of Power (ICHQP), pp. 1-6 (2010). doi:10.1109/ICHQP.2010.5625499

30. Ortmeyer, T.H., Reeves, J.A., Hou, D., McGrath, P.: Evaluation of sustained and momentary interruption impacts in reliability-based distribution system design. IEEE Trans. Power Deliv. 25(4), 31333138 (2010). doi:10.1109/TPWRD.2010.2052075

31. Reliability of Electric Utility Distribution Systems: EPRI White Paper, EPRI, Palo Alto, CA: 2000. 1000424 (2006)

32. Sattler, C., Stachow, U., Berger, G.: Expert knowledge-based assessment of farming practices for different biotic indicators using fuzzy logic. J. Environ. Manag. 95(1), 132-143 (2012)

33. Tsai, R.S.R.S.: Reliability worth guides distribution system design. IEEE Trans. Ind. Appl. I(4), 368-375 (1979)

34. Wangdee, W., Billinton, R.: Reliability-performance-index probability distribution analysis of bulk electricity systems. Can. J. Electr. Comput. Eng. 30(4), 189-193 (2005). doi:10.1109/CJECE.2005. 1541750

35. Warren, C.A.: Distribution reliability: what is it? IEEE Ind. Appl. Mag. 2(4), 32-37 (1996). doi:10.1109/2943.503526

36. Willis, H.L.: Power Distribution Planning Reference Book. CRC Press, Boca Raton (2010)

37. Zadeh, L.A., Klir, G.J., Yuan, B.: Fuzzy Sets, Fuzzy Logic, and Fuzzy Systems: Selected Papers, vol. 6. World Scientific, Singapore (1996) 